\title{
Interactions between Stem Cells and Their Niche in the Drosophila Ovary
}

\author{
T. Xie, X. Song, Z. Jin, L. Pan, C. Weng, S. Chen, And N. Zhang \\ Stowers Institute for Medical Research, Kansas City, Missouri 64110
}

\begin{abstract}
The Drosophila ovary contains at least three types of active stem cells, namely, germ-line stem cells (GSCs), escort stem cells (ESCs), and follicular stem cells (FSCs), which work together to efficiently assemble egg chambers. Among the three stem cell types, the GSC is among the first shown to be controlled by the niche due to its easy identification and welldefined surrounding cells. We have shown that the niche controls GSC self-renewal, anchorage, aging, and competition, and the GSC also signals back to the niche for its maintenance. The FSC is an attractive model for studying epithelial stem cell regulation and signal integration because we have shown that it resembles mammalian epithelial stem cells and requires multiple signaling pathways for its self-renewal. In this chapter, we have highlighted the findings of our studies on interactions between Drosophila ovarian stem cells and their niches during normal development and aging and on stem cell competition for niche occupancy. We further discuss their implications in general stem cell biology and future directions in this exciting area.
\end{abstract}

Stem cell self-renewal and differentiation have been shown to be controlled by the regulatory microenvironment or niche in many different systems ( $\mathrm{Li}$ and Xie 2005; Morrison and Spradling 2008). However, it remains largely unclear how niche signals collaborate with intrinsic factors to control stem cell self-renewal and differentiation. In the past 8 years, our laboratory has been using Drosophila ovary as a model system to identify niche signals and intrinsic factors that are essential for stem cell self-renewal and to study the molecular mechanisms underlying stem cell/niche interactions, niche formation and maintenance, stem cell aging, and competition.

Each Drosophila female carries 30-40 individual egg assembly lines known as ovarioles, where the anterior tip has the germarium in which three types of active stem cells reside (Fig. 1A,B). The terminal filament (TF) is positioned at the most anterior end of the germarium, whereas the cap cells, located posterior to the TF, contact GSCs posteriorly and ESCs laterally (Fig. 1B-D). GSCs can be identified by their location (in contact with cap cells) and anteriorly localized spectrosome, which can be labeled with Hts staining (Fig. 1C,D). We have shown that cap cells are necessary and sufficient for maintaining GSCs (Xie and Spradling 2000; Song et al. 2007). Interestingly, when both of the daughters of a GSC are in contact with cap cells, they both become stem cells, representing the first demonstration of the existence of the stem cell niche (Xie and Spradling 2000). Consistently, cap cells express $\mathrm{Yb}$ and Piwi, which are required for maintaining GSCs (Cox et al. 1998, 2000; King and Lin 1999; King et al. 2001). In addition, cap cells also express secreted bone morphogenetic protein (BMP)-like growth factors, which are essential for GSC self-renewal (Xie and Spradling 1998, 2000; Song et al. 2004). Intrinsically, two
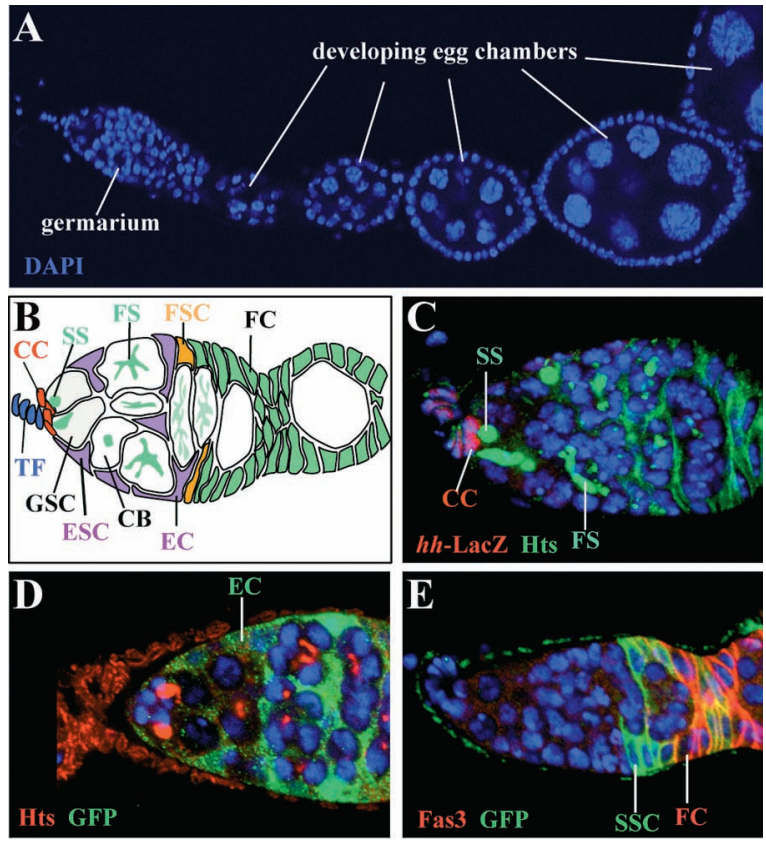

Figure 1. Structures of Drosophila ovariole and germarium. $(A)$ A part of an ovariole, which is stained by DAPI (blue), contains a germarium and five developing egg chambers. $(B)$ Diagram of a germarium: TF (terminal filament, blue); CC (cap cell, red); ESC (escort stem cell, purple); EC (escort cell, purple); SS (spectrosome, green); FS (fusome, green); FSC (follicular stem cell, brown); and FC (follicle cell, green). (C) A germarium, labeled for LacZ (TF and CCs, red) and Hts (SS and FS, green), shows cap cells and two GSCs. (D) A part of a germarium, labeled for Hts (red) and GFP (green), shows the escort cells (green). (E) A germarium, labeled for Fas3 (FCs, red) and GFP (green), shows a GFP-marked FSC clone. 
classes of intrinsic factors control the balance between GSC self-renewal and differentiation: self-renewing factors such as Pumilio and Nanos and differentiation-promoting factors such as Bam and Bgen (McKearin and Spradling 1990; Lin and Spradling 1997; Forbes and Lehmann 1998; Ohlstein et al. 2000; Wang and Lin 2004). The interplay between niche signals and intrinsic factors that control Drosophila ovarian GSCs has just begun to be revealed (Chen and McKearin 2003a; Song et al. 2004; Xi and Xie 2005).

Two FSCs, previously also known as somatic stem cells (SSCs), which can be followed by green fluorescent protein (GFP) or LacZ markers, are located on the opposite sides of the germarium to produce the follicular epithelium that wraps around germ cell cysts produced by GSCs (Fig. 1E) (Margolis and Spradling 1995; Zhang and Kalderon 2001; Song and Xie 2002; Nystul and Spradling 2007). Cap-cell-expressing Hedgehog (Hh) is required for maintaining FSC self-renewal (Forbes et al. 1996; King et al. 2001; Zhang and Kalderon 2001). Using genetically marked mutant FSCs, we have shown that cap-cellexpressing Wingless and escort-cell-expressing BMP-like Gbb directly control FSC self-renewal (Song and Xie 2003; Kirilly et al. 2005). In addition, E-cadherinmediated cell adhesion is also required for maintaining FSCs by keeping them in the proximity of signaling resources (Song and Xie 2002). The adhesion may help early FSC progeny to migrate from one side of the germarium to the other for stem cell replacement (Nystul and Spradling 2007). In this chapter, we summarize the findings from our studies on Drosophila ovarian GSCs and FSCs and discuss their potential implications in other stem cell systems.

\section{SHORT-RANGE BMP NICHE SIGNALS \\ CONTROL GSC SELF-RENEWAL BY DIRECTLY REPRESSING DIFFERENTIATION}

Although it is known that Dpp (Decapentaplegic)/BMP is a niche-derived growth factor essential for GSC selfrenewal (Xie and Spradling 1998), it remains unclear how this BMP-like factor mechanistically controls stem cell self-renewal and whether other BMP-like molecules also participate in GSC regulation. We have found that similar to $d p p$, another Drosophila BMP-like gene $g b b$ is expressed specifically in the somatic cells surrounding GSCs, including cap cells, and $g b b$ mutant females lose their GSCs prematurely, indicating that Gbb is also an essential niche signal (Song et al. 2004). To further our understanding of how BMP-like signals control GSC selfrenewal, we have shown that only GSCs, but not cystoblasts (immediate differentiating GSC daughters lying one cell away from cap cells), express pMad and Dad, two BMP activity indicators, indicating that BMPs only function in one cell diameter (Fig. 2A) (Song et al. 2004). Expressed in cystoblasts, bam is necessary and sufficient for their differentiation because mutations in bam can completely block cystoblast differentiation, and its forced expression in GSCs can sufficiently cause their differentiation (McKearin and Ohlstein 1995; Ohlstein and McKearin 1997). Although it is normally repressed in wild-type GSCs, bam transcription is up-regulated in the GSCs that are in the $d p p$ and $g b b$ mutant niche or are mutant for BMP downstream transducers, indicating that BMP signaling is essential for repressing bam expression and thus maintaining GSC self-renewal (Fig. 2C,D) (Song et al. 2004). Dpp overexpression can completely repress
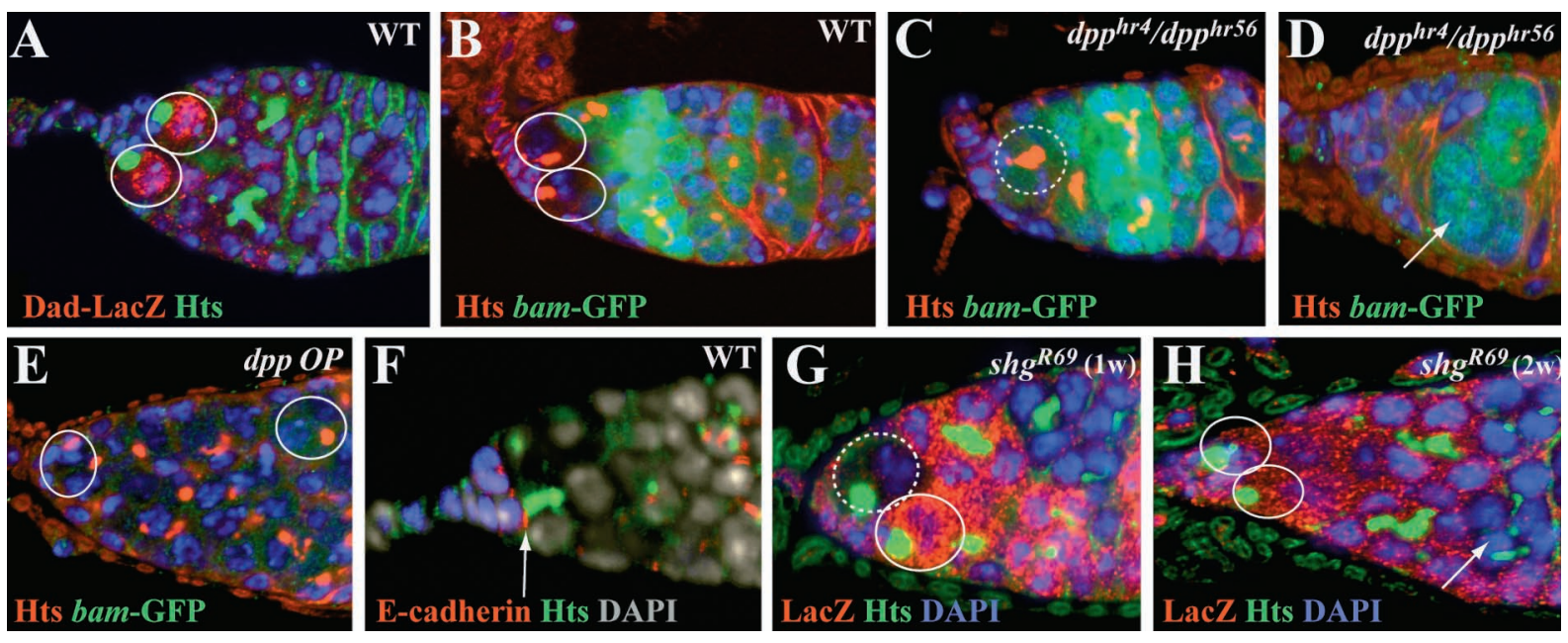

Figure 2. Niche-derived BMP signaling and E-cadherin-mediated GSC-niche adhesion are required for GSC maintenance. (A) A germarium shows the restricted BMP signaling activity indicated by Dad-lacZ expression (red) in GSCs (solid circles). (B) A germarium shows the repression of bam-GFP expression (green) in GSCs (solid circles). (C) A dpp mutant germarium shows the up-regulated expression of bam-GFP (green) in the remaining GSC (dashed circle). (D) A dpp mutant germarium shows the differentiation of a bam $G F P$-expressing GSC into a cyst (arrow) and thus GSC loss. (E) The tip of a $d p p$-overexpressing ( $d p p O P$ ) germarium shows the absence of bam-GFP expression (green) in single germ cells (circles). (F) The tip of a wild-type germarium shows the accumulation of E-cadherin $(r e d)$ in the GSC-niche junction (arrow). $(G)$ The tip of a wild-type germarium carries one marked week-old shg mutant GSC and an unmarked wild-type GSC. $(H)$ The tip of a wild-type germarium carries two unmarked wild-type GSCs and marked shg mutant cysts, indicating the loss of a marked mutant GSC (near arrow). 
bam expression in germ cells by activating and forming the Mad-Medea transcriptional complex, which can directly bind to a bam silencer (Fig. 2E) (Chen and McKearin 2003a,b; Song et al. 2004). Similarly, we have demonstrated that niche-derived BMP signaling controls GSC self-renewal by repressing bam expression in the Drosophila testis (Kawase et al. 2004). Therefore, BMP signals maintain the self-renewal of GSC daughters remaining in the niche by directly repressing bam expression and allow other daughters outside the niche to differentiate due to their short-range function. These findings have, for the first time, offered a simple model to explain the stem cell dogma: A stem cell divides to generate a selfrenewing stem cell in the niche and a differentiating daughter staying out of the niche.

\section{E-CADHERIN-MEDIATED CELL ADHESION ANCHORS GSCS IN THE NICHE FOR THEIR SELF-RENEWAL}

Because BMP niche signals only function as one cell diameter, the temporary departure of GSCs from the niche can potentially jeopardize their self-renewal potential. Thus, it is imperative to know how self-renewing GSCs are maintained in the niche constantly. We have shown that E-cadherin and its associated protein Armadillo ( $\beta$ catenin) are expressed in GSCs and cap cells and accumulated in the stem cell/niche junction to form adherens junctions, indicating that GSCs are anchored to their niche (Fig. 2F) (Song et al. 2002). Removal of E-cadherin or Armadillo specifically from GSCs themselves sufficiently disrupts adherens junctions between GSCs and cap cells due to homophilic interactions and causes rapid GSC loss, further indicating that the niche anchorage through E-cadherin-mediated cell adhesion is essential for GSC self-renewal (Fig. 2G,H). In addition, E-cadherin is required for recruiting GSCs to their niche during niche and GSC formation (Song et al. 2002). Therefore, these findings demonstrate that E-cadherin-mediated niche anchorage is important for GSC maintenance (Song et al. 2002). Intriguingly, N-cadherin has been shown to be required for keeping mouse hematopoietic stem cells in the niche, indicating that cadherin-mediated stem cell anchorage is a conserved mechanism for anchoring stem cells in the niche (Haug et al. 2008).

\section{INTRINSIC FACTORS ARE ALSO REQUIRED FOR CONTROLLING GSC SELF-RENEWAL BY REPRESSING DIFFERENTIATION}

Although we have shown that BMP signaling can directly repress bam expression in GSCs and thereby maintain self-renewal, it remains unclear how the BMP-regulated Medea-Mad (SMAD) transcriptional complex represses bam transcription, because SMADs are known to be transcriptional activators in mammals (Kretzschmar and Massagué 1998). We have recently shown that an ATPdependent chromatin-remodeling protein ISWI, a member of the SNF2/SWI protein family, is required in GSCs for their self-renewal, because marked mutant iswi GSCs are rapidly lost from the niche (Fig. 3A) (Xi and Xie 2005).
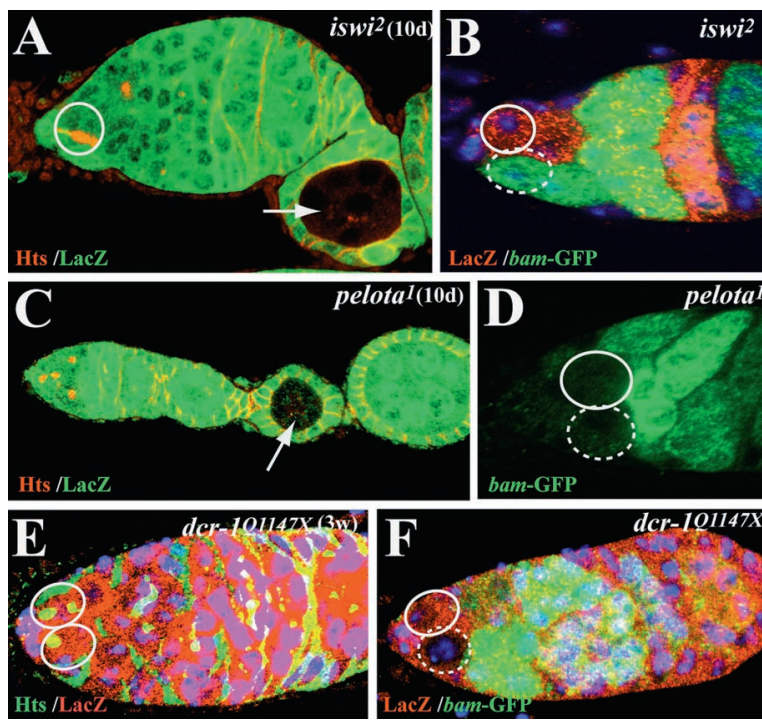

Figure 3. Intrinsic factors ISWI, Pelota, and Dcr-1 are required for GSC self-renewal. (A) A germarium only contains wild-type GSCs (solid circle) with the marked iswi mutant GSC differentiating into a cyst in an egg chamber (arrow). (B) bam-GFP expression is up-regulated in the marked iswi mutant GSC (broken circle) but not in the unmarked wild-type GSC (solid circle). (C) A part of an ovariole shows that the lost marked pelota mutant GSC has differentiated into a cyst in an egg chamber (arrow). (D) In the unmarked wild-type GSC (solid circle) and the marked pelota mutant GSC (dashed circle), bam-GFP expression is still repressed. (E) A germarium only contains wild-type GSCs (solid circles) with a lost marked dcr-1 mutant GSC moving out of the germarium (not shown). $(F)$ In the marked $d c r-1$ mutant GSC and the unmarked wild-type GSC, bam-GFP expression remains repressed.

Interestingly, in the iswi mutant GSCs, bam transcription is elevated, and BMP signaling activity is down-regulated, indicating that ISWI is required for BMP-signalingmediated transcriptional repression for bam in GSCs (Fig. 3B). Therefore, this study demonstrates that chromatin remodeling or epigenetic control has a critical role in controlling GSC self-renewal (Xi and Xie 2005). Because ISWI is a catalytic subunit of three different chromatinremodeling complexes, namely, CHRAC (chromatin accessibility complex), NURF (nucleosome-remodeling factor), and ACF (ATP-utilizing chromatin assembly and remodeling factor) (Deuring et al. 2000), it would be interesting to investigate which ISWI-containing complex is involved in GSC regulation and if and how the ISWI-containing complex interacts with the Mad-Medea complex in repressing bam expression.

To further understand how BMP signaling controls GSC self-renewal, pelota (pelo), a gene known only to be required for Drosophila male meiosis (Eberhart and Wasserman 1995), was identified genetically as a dominant suppressor of the $d p p$ overexpression-induced GSC tumor phenotype (Xi et al. 2005). In pelo mutant ovaries, GSCs are lost rapidly owing to differentiation, and our genetic results show that it functions inside GSCs to control self-renewal (Fig. 3C). In those pelo mutant GSCs, bam expression is still repressed, and bam mutant germ 
cells are still able to differentiate into cystocytes without pelo function, indicating that pelo controls GSC selfrenewal by repressing a bam-independent differentiation pathway (Fig. 3D). Because Pelo is shown to function as an RNA endonuclease (Lee et al. 2007), our findings suggest that Pelo is involved in degrading mRNAs encoding proteins important for GSC differentiation (Xi et al. 2005). It is important to identify Pelo target mRNAs in GSCs to obtain a better understanding of how Pelo controls GSC self-renewal. Because Pelo is highly conserved from Drosophila to mammals, it may also be involved in the regulation of adult stem cell self-renewal in mammals, including humans.

microRNAs (miRNAs) regulate gene expression to modulate a variety of cellular events such as cell-fate determination and maintenance by controlling the turnover and/or translation of specific mRNAs in different cell types and organisms (Valencia-Sanchez et al. 2006). In Drosophila, Dicer-1 (Dcr-1) and Loquacious (Loqs) form a protein complex essential for generating mature miRNAs from their corresponding precursors. In the Drosophila ovary, Dcr-1 was first shown to be required for controlling GSC division only (Hatfield et al. 2005). Surprisingly, our results show that $d c r-1$ mutant GSCs are lost rapidly from the niche without discernible features of cell death, indicating that Dcr-1 controls GSC selfrenewal (Fig. 3E) (Jin and Xie 2007). bam transcription and protein expression, however, are not up-regulated in $d c r-1$ mutant GSCs, and their removal does not slow down dcr-1 mutant GSC loss, suggesting that Dcr-1 controls GSC self-renewal by repressing a Bam-independent differentiation pathway (Fig. 3F) (Jin and Xie 2007). In addi- tion, Loqs is also shown to be required in GSCs for their maintenance (Park et al. 2007). Therefore, the miRNA pathway is required not only for regulating the GSC division rate, but also for controlling GSC self-renewal. In the future, it will be exciting to investigate functions of individual miRNAs and their targets in GSC regulation.

\section{NOTCH SIGNALING CONTROLS GSC-NICHE FORMATION AND MAINTENANCE}

Although a number of stem cell niches have been defined, it remains largely unknown how niche formation and maintenance are controlled. As mentioned earlier, cap cells are a key cellular component of the GSC niche (Xie and Spradling 2000). During the late third-instar larval stage, the TFs in the developing female gonad form before the emergence of cap cells (Zhu and Xie 2003). Interestingly, these newly formed TF cells express high levels of Delta that can only activate Notch signaling in adjacent Notch-expressing somatic precursor cells in the posterior because Delta is a transmembrane ligand (Song et al. 2007). Indeed, Notch signaling, detected by an E(spl) reporter line, is active in the cells that lie adjacent to the TF posteriorly and are destined to form cap cells (Fig. 4A) (Song et al. 2007). Expanded Notch activation causes the formation of more cap cells, which support more GSCs, whereas compromising Notch signaling during niche formation decreases the cap cell number and consequently the GSC number (Fig. 4B) (Song et al. 2007). Furthermore, the niches located away from their normal location can still sufficiently sustain GSC self-renewal by maintaining high local BMP signaling and repressing bam in
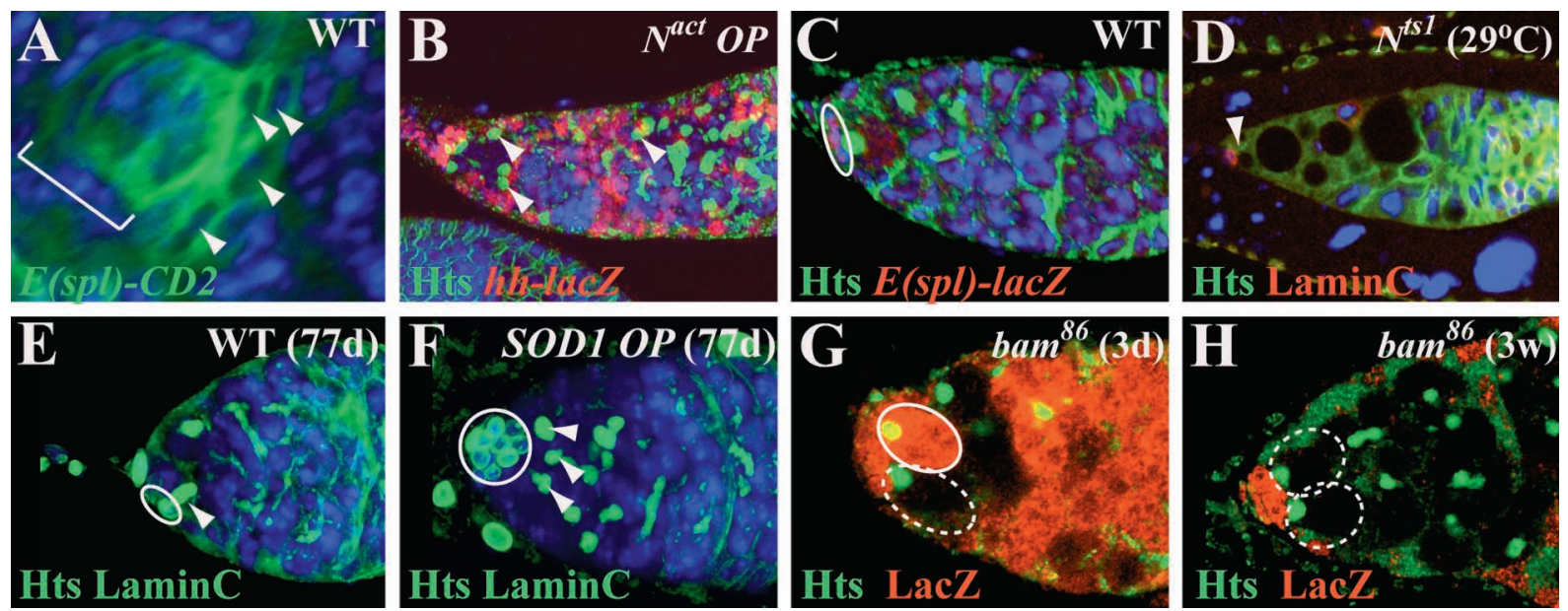

Figure 4. Notch signaling controls the maintenance and formation of the GSC niche, which further contributes to GSC aging and competition. $(A)$ In the larval-pupal transitional stage, newly formed TFs (bracket) and cap cells (arrowheads) have active Notch signaling indicated by $E(s p l)-C D 2$ expression (green). (B) Ectopic activation of Notch signaling during the third-instar larval stage can lead to formation of excessive cap cells (red) that in turn support more GSCs (arrowheads). (C) The cap cells (oval) in the adult ovary retain active Notch signaling activity indicated by $E(\mathrm{spl}$-lacZ (red). (D) A germarium (its tip indicated by arrowhead) shows the loss of cap cells and GSCs 2 weeks after inactivation of Notch signaling. (E) A 77-day-old germarium carries two cap cells (oval) and one GSC (its spectrosome indicated by arrowhead). (F) A 77-day-old germarium, in which SOD1 is overexpressed in the cap cells, still has eight cap cells (circle) and three GSCs (arrowheads), like a young germarium. $(G)$ A germarial tip contains unmarked wild-type GSC (circle) and a 3-day-old marked bam mutant GSC (dashed circle). (H) A germarial tip contains two marked bam mutant GSCs (dashed circles) 3 weeks after generation of a marked GSC. In the same ovariole, the outcompeted wild-type GSC has differentiated into a normal cyst in an old egg chamber. 
ectopic GSCs. Similar findings on formation of extra cap cells induced by expanded Notch signaling have also been made by the Ruohola-Baker group (Ward et al. 2006). Finally, Delta starts to gain its expression in adult GSCs and maintains active Notch signaling in the cap cells of the adult ovary, and loss of Notch function results in rapid loss of the adult GSC niche, including cap cells (Fig. 4C,D). These findings demonstrate that Notch signaling is important for formation and maintenance of the GSC niche and that cap cells help to determine niche size and function (Song et al. 2007).

We have also shown that following the niche formation, anterior primordial germ cells (PGCs) adjacent to cap cells can develop into GSCs at the early pupal stage while the rest differentiate directly (Zhu and Xie 2003). The anterior PGCs are very mitotically active and exhibit two division patterns with respect to the niche: asymmetric division (one daughter contacting cap cells and the other daughter away from cap cells) and symmetric division (both daughters contacting cap cells). Indeed, our genetic lineage analysis results indicate that newly formed GSCs can divide asymmetrically and symmetrically (Zhu and Xie 2003). At the third-instar larval stage, $d p p$ overexpression promotes PGC proliferation and causes the accumulation of more PGCs in the gonad. The PGCs mutant for $t k v$, encoding an essential $d p p$ receptor, lose their ability to divide symmetrically. Therefore, $d p p$ is probably one of the mitotic signals that promote the symmetric division and expansion of GSCs during niche and GSC formation (Zhu and Xie 2003).

\section{NICHE AGING AND INTRINSIC GSC AGING COLLECTIVELY CONTRIBUTE TO OVERALL GSC AGING}

It is widely postulated that tissue aging could be, at least partially, caused by reduction of stem cell number, activity, or both. However, it largely remains a mystery as to how stem cells and their niche deal with the aging process. Indeed, the number of GSCs and cap cells in old Drosophila females decreases in comparison with that in young females, and the GSC proliferation rate also declines with age (Fig. 4E) (Pan et al. 2007). As expected, BMP signaling activity and E-cadherin accumulation in the stem cell/niche junction undergo an age-dependent decline. In females heterozygous for mutations in the BMP signaling and E-cadherin-mediated adhesion pathways, the age-dependent decline in the number of GSCs and cap cells is accelerated, whereas providing more BMP in the niche or E-cadherin in GSCs can significantly rescue the age-dependent decline in GSC number and proliferation rate, demonstrating that the age-dependent decline in BMP signaling and E-cadherin-mediated cell adhesion contribute to GSC aging (Pan et al. 2007).

Reactive oxygen species (ROS)-induced cellular damage is known to cause cellular and organismal aging, whereas overexpression of superoxide dismutase (SOD), an enzyme that helps remove ROS from the cell, can prolong life span in Drosophila (Tower 2000). Interestingly, overexpression of SOD specifically in cap cells can prolong the life span of cap cells and GSCs and promote proliferation of aged
GSCs, indicating that the aged niche contributes to overall stem cell aging (Fig. 4F) (Pan et al. 2007). Similarly, SOD overexpression in GSCs alone can prolong GSC life span and promote GSC proliferation, indicating that GSCs also undergo intrinsic aging. Therefore, our study demonstrates that ROS-induced cellular damage causes niche aging and intrinsic stem cell aging, which collectively contribute to overall stem cell aging (Pan et al. 2007).

\section{COMPETITION IS USED BY DIFFERENTIATION-DEFECTIVE STEM CELLS FOR THEIR EXPANSION AND MAY ALSO SERVE AS A STEM CELL QUALITY CONTROL MECHANISM}

Although much progress has recently been made regarding how the niche controls stem cell function, little is yet known about how stem cells in the same niche or tissue interact with one another. In addition, cancer stem cells (CSCs) have recently been proposed to be rare, selfrenewing mitotic cells for driving tumor growth, but it remains unclear how CSCs interact with normal stem cells. We have used Drosophila ovarian GSCs as a model system to investigate how stem cells interact with one another in the same niche or tissue. bam or bgcn mutant GSCs can continuously self-renew and generate a large number of differentiation-defective stem cells, resembling mammalian CSCs (McKearin and Ohlstein 1995; Kai et al. 2005). The differentiation-defective bam or bgcn mutant GSCs gradually push neighboring wild-type GSCs out of the niche, indicating that those CSC-like bam or bgcn mutant GSCs are more competitive than wild-type ones (Fig. 4G,H) (Jin et al. 2008). Furthermore, bam or bgcn mutant GSCs up-regulate E-cadherin expression in the stem cell/niche junction, and different E-cadherin levels can sufficiently stimulate GSC competition (Jin et al. 2008). Therefore, our findings demonstrate that differentiation-defective stem cells can outcompete normal stem cells for niche occupancy through up-regulation of E-cadherin and further suggest that CSCs may use competition to expand themselves and invade normal tissues (Jin et al. 2008).

What is the biological function of competition in stem cell regulation? We have shown that when GSCs differentiate and up-regulate bam expression, they are detached from the niche and are lost rapidly, although there are adherens junctions between GSCs and their niche (Song et al. 2002, 2004; Xi and Xie 2005). Stem cell competition offers important insight into how differentiated stem cells are expelled out of the niche. The following is our current working model: A differentiating GSC up-regulates bam expression and consequently down-regulates E-cadherin expression; over time, the mutant GSC has less E-cadherin than its wild-type neighboring stem cell in the same niche and is then pushed out of the niche by a wild-type neighboring stem cell, which then generates a new stem cell to replace the lost one. Thus, we propose that GSCs have a competitive relationship for niche occupancy, which likely serves as a quality control mechanism to ensure that accidentally differentiated stem cells are rapidly removed from the niche and replaced by functional ones. 


\section{FSCS CONTROL THEIR SELF-RENEWAL USING A DIFFERENT COMBINATION OF NICHE SIGNALS FROM GSCS}

To investigate if and how different stem cell types in the same tissue are regulated differently, we have chosen to study FSC regulation in the Drosophila ovary. We have shown that Wingless $(\mathrm{Wg})$ is also expressed in cap cells and is required for follicle cell production (Song and Xie 2003). Down-regulation of Wg signaling through removal of its positive regulators disheveled $(d s h)$ and armadillo $(\mathrm{arm})$ results in rapid FSC loss (Fig. 5A,B). Surprisingly, constitutive Wg signaling in FSCs through the removal of its negative regulators Axin and shaggy also causes their loss, suggesting that appropriate levels of $\mathrm{Wg}$ signaling are critical for FSC self-renewal (Song and Xie 2003). In addition, constitutive Wg signaling causes overproliferation and abnormal differentiation of early FSC progeny. These findings demonstrate that $w g$ signaling regulates FSC maintenance and also influences follicle cell differentiation (Song and Xie 2003). In mammals, Wnt signaling is important for maintaining the epithelial stem cell compartment in the intestine (Korinek et al. 1998; Alonso and Fuchs 2003), whereas constitutive Wnt signaling causes overproliferation and abnormal differentiation of epithelia stem cells in the skin and the intestine, resulting in cancer formation (Korinek et al. 1997; DasGupta and Fuchs 1999). Possibly, mechanisms regulating proliferation and differentiation of epithelial stem cells are conserved from Drosophila to humans.

$d p p$ is restricted to cap cells and follicle cells, whereas $g b b$ is expressed in all of the somatic cells including cap cells and escort cells covering differentiated germ cells, but their role in FSC regulation remains unclear (Song et al. 2004). We have shown that although $d p p$ mutant ovaries show a weak follicle cell production defect, $g b b$ mutant ovaries exhibit a severe deficiency in follicle cell production (Kirilly et al. 2005). In addition, the marked
FSC clones mutant for BMP downstream components have retarded proliferation and are lost rapidly, indicating that BMP signaling is necessary for promoting FSC selfrenewal and proliferation (Fig. 5C,D). Moreover, constitutive BMP signaling prolongs the FSC life span. Therefore, BMP signaling directly promotes SSC self-renewal and proliferation in the Drosophila ovary (Kirilly et al. 2005). In the future, it will be critical to understand how these different signaling pathways are integrated in FSCs to control their self-renewal.

As one would have expected, FSCs must stay close to sources for self-renewing signals, including cap cells and escort cells, in order to maintain their self-renewal. We have shown that E-cadherin-mediated cell adhesion is required for keeping FSCs in the proximity to signaling sources for their self-renewal (Song and Xie 2002). The marked shg or arm mutant FSC clones are rapidly lost from germaria (Fig. 5E). In addition, we have identified Domino (Dom), an SNF2-related ATP-dependent chromatin remodeling factor, as an essential intrinsic factor for controlling FSC self-renewal because the marked dom mutant FSCs are lost rapidly from germaria (Fig. 5F) (Xi and Xie 2005). Finally, we have also shown that the miRNA pathway is needed in FSCs for their self-renewal (Jin and Xie 2007). In the future, it will be of great interest to study the relationships between intrinsic pathways and niche-signal-mediated signaling pathways.

\section{CONCLUSIONS AND FUTURE DIRECTIONS}

Along with the findings from other colleagues in the field, our findings on Drosophila ovarian GSC and FSC regulation have produced several general principles for stem cell biology as described below.

First, the stem cell is anchored to its niche through cadherin-mediated cell adhesion to ensure long-term selfrenewal. Both GSCs and FSCs require E-cadherin-mediated
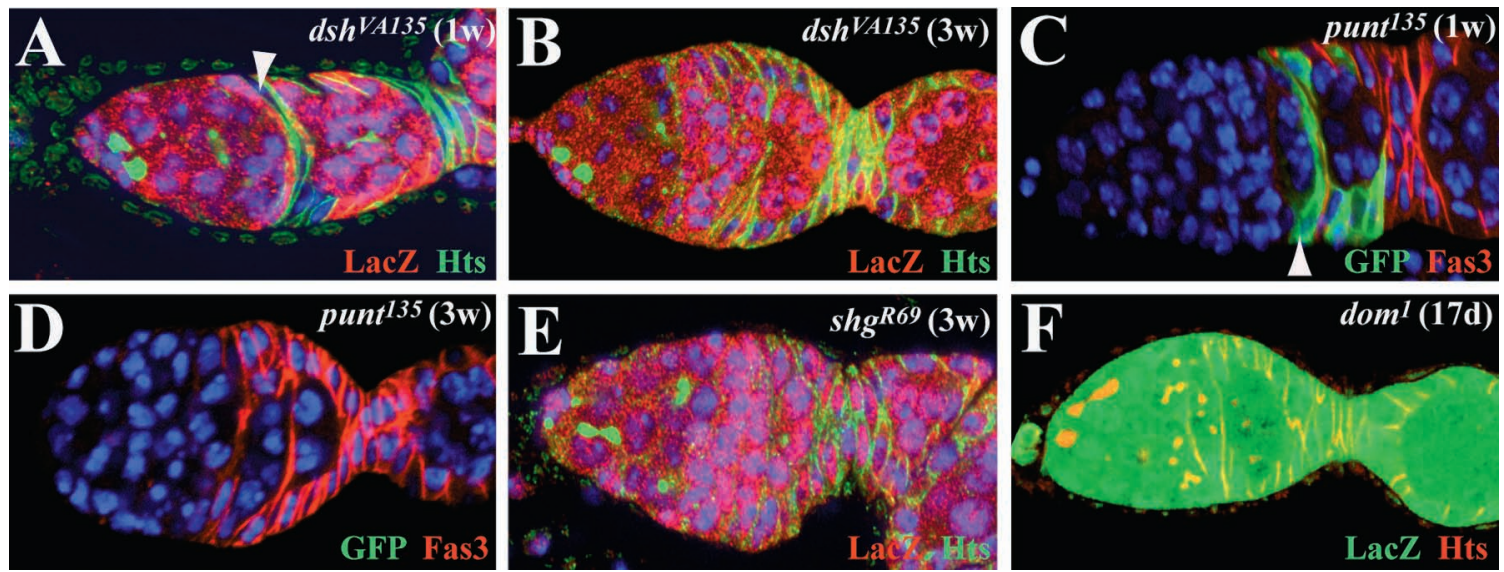

Figure 5. Wingless signaling, BMP signaling, E-cadherin-mediated adhesion, and Dom are required for maintaining FSCs. $(A)$ A germarium carries a LacZ-negative marked $d s h$ mutant FSC (arrowhead) 1 week after clone induction. (B) A germarium has lost a LacZnegative marked $d s h$ mutant FSC clone 3 weeks after clone induction. (C) A germarium carries a GFP-positive marked punt mutant FSC (arrowhead) 1 week after clone induction. punt encodes a BMP type II receptor. $(D)$ A germarium has lost a GFP-positive marked punt mutant FSC clone 3 weeks after clone induction. $(E)$ A germarium has lost a LacZ-negative marked shg mutant FSC clone 3 weeks after clone induction. (E) A germarium has lost a LacZ-negative marked dom mutant FSC clone 3 weeks after clone induction. 
cell adhesion to keep them in the proximity of signals from the niche (Fig. 6) (Song and Xie 2002; Song et al. 2002). Such anchorage guarantees that the future stem cell will continuously self-renew and that the unanchored daughter that leave the niche and differentiate.

Second, different stem cell niches can have distinct structures and use different combinations of signals (Fig. 6). The GSC niche is composed of cap cells and possibly ESCs, and produces BMPs and the unidentified Piwi-regulated signal controlling GSC self-renewal and proliferation (Xie and Spradling 1998, 2000; King et al. 2001; Song et al. 2004, 2007; Decotto and Spradling 2005), whereas the FSC niche is composed of at least cap cells and escort cells and produces BMP, Hh, and Wg signals for promoting self-renewal and proliferation (Forbes et al. 1996; Zhang and Kalderon 2001; Song and Xie 2003; Kirilly et al. 2005). Interestingly, cap cells produce self-renewing signals for both GSCs and FSCs, likely representing a common niche component for coordinating the activities of both stem cells. In addition, BMPs from cap cells only function in one cell diameter to control GSC self-renewal (Kai and Spradling 2003; Song et al. 2004), whereas $\mathrm{Wg}$ and $\mathrm{Hh}$ in cap cells function in many cell diameters to control FSC self-renewal (Forbes et al. 1996; King et al. 2001; Song and Xie 2003).

Third, different classes of intrinsic factors are required for GSC or FSC self-renewal (Fig. 6). In addition to previously identified Pumilio/Nanos, cyclin B, and Stonewall (Lin and Spradling 1997; Forbes and Lehmann 1998; Wang and Lin 2005; Maines et al. 2007), we have shown that Dcr1, Pelota, and ISWI are required intrinsically for GSC selfrenewal, and Dcr-1 and Dom are required intrinsically for FSC self-renewal (Xi et al. 2005; Xi and Xie 2005; Jin and Xie 2007). These intrinsic factors are involved in different biological pathways, such as mRNA translation and degradation, chromatin remodeling, miRNAs, and the cell cycle.
Fourth, intrinsic factors and niche signals control selfrenewal by repressing expression or functions of differentiation-promoting genes. BMP niche signaling, Piwimediated niche signaling (Fig. 6), and ISWI repress expression of the differentiation-promoting gene bam in GSCs and thus maintain their undifferentiated state (Chen and McKearin 2003a, 2005; Song et al. 2004; Szakmary et al. 2005; Xi and Xie 2005), whereas the intrinsic factors Pumilio/ Nanos, Pelota, Stonewall, and Dcr-1/Loqs are required for maintaining GSC self-renewal by repressing a bam-independent pathway (Gilboa and Lehmann 2004; Wang and Lin 2004; Xi et al. 2005; Jin and Xie 2007; Maines et al. 2007; Park et al. 2007).

Fifth, the stem cell niche requires signals for maintenance from its resident stem cells (Fig. 6). We have shown that Notch signaling is required for GSC-niche formation during early development, and GSC-expressing Delta can signal back to cap cells for their maintenance and integrity in the adult ovary (Ward et al. 2006; Song et al. 2007). These findings indicate that stem cells and their niche are mutually dependent.

Sixth, stem cells and their niche undergo intrinsic aging, collectively contributing to overall stem cell aging (Fig. 6). ROS-induced cellular damage leads to niche and GSC aging in the Drosophila ovary (Pan et al. 2007).

Finally, competition serves as a quality control mechanism. We have found that bam and bgcn mutant GSCs can outcompete wild-type GSCs by up-regulating E-cadherin expression (Jin et al. 2008). This finding also suggests the existence of a quality control mechanism to ensure that only undifferentiated stem cells stay in the niche. So far, some of the generalizations of the control mechanisms for Drosophila ovarian stem cells have been confirmed and also have provided guidance for studying stem cells in mammalian systems; others await further verification.
A

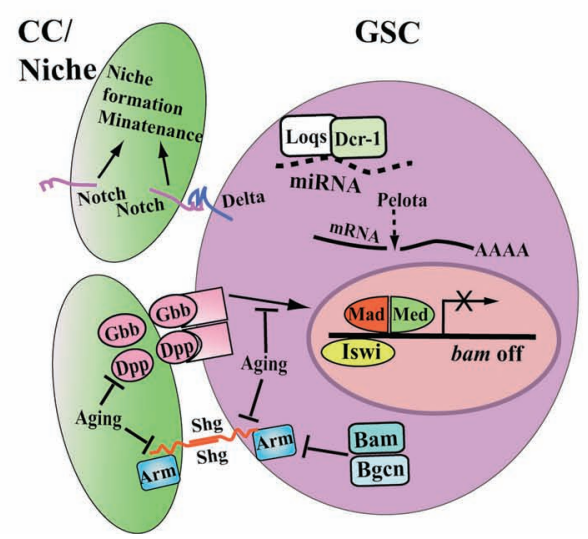

B

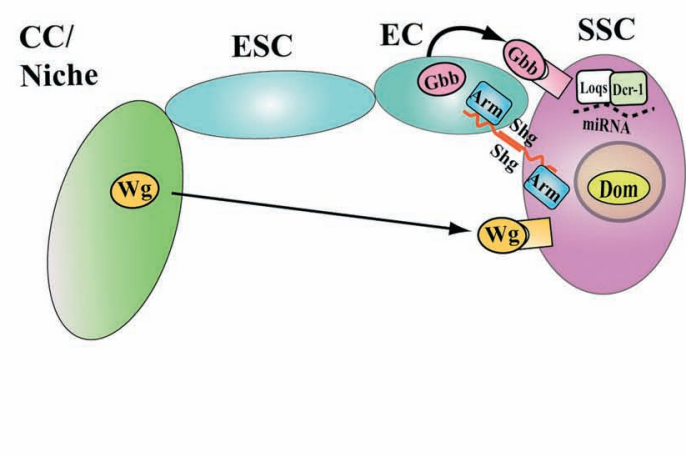

Figure 6. Schematic diagrams summarizing our contributions to understanding Drosophila ovarian GSC and FSC regulation. (A) For GSCs, we have identified niche-derived BMP signaling for maintaining self-renewal, E-cadherin for anchorage in the niche, Notch signaling for controlling niche formation and maintenance, intrinsic factors Dcr-1, Pelota, and ISWI for controlling self-renewal, and $\mathrm{Bam} / \mathrm{Bgcn}$ for controlling stem cell competitiveness. In addition, we have also shown that ROS-induced cellular damage leads to niche aging and intrinsic GSC aging, collectively contributing to overall GSC aging. (B) For FSCs, we have identified BMP and Wingless signaling pathways and chromatin remodeling factors Dom and Dcr-1 for controlling self-renewal and E-cadherin for anchorage in the niche. 
Although much progress has been made in studying Drosophila ovarian stem cells, such as defining stem cell niche structures and signals and identifying self-renewalpromoting intrinsic factors, many important questions still remain to be answered. In the future, we will continue to use a combination of genetic, molecular, biochemical, genomic, and cell biological approaches and Drosophila ovarian stem cells as a model system to address the following important stem-cell-related questions, including: How are GSC and FSC niches built in the Drosophila ovary? How do shared niche components contribute to functions of different niches and coordination of these stem cell activities? What are additional intrinsic factors and extrinsic signals from the niche for controlling GSC and FSC self-renewal? How are multiple pathways integrated in stem cells to control self-renewal? How do intrinsic factors interact with extrinsic signals to control stem cell self-renewal, proliferation, and differentiation? The answers to these questions will provide further insights into how Drosophila ovarian stem cells are regulated and how stem cells are controlled in general.

\section{ACKNOWLEDGMENTS}

Our special thanks go to all the former Xie laboratory members for their outstanding scientific contributions. The work in the Xie lab is supported by the Stowers Institute for Medical Research and the National Institutes of Health (GM64428).

\section{REFERENCES}

Alonso, L. and Fuchs, E. 2003. Stem cells in the skin: Waste not, Wnt not. Genes Dev. 17: 1189-1200.

Chen, D. and McKearin, D. 2003a. Dpp signaling silences bam transcription directly to establish asymmetric divisions of germline stem cells. Curr. Biol. 13: 1786-1791.

Chen, D. and McKearin, D.M. 2003b. A discrete transcriptional silencer in the bam gene determines asymmetric division of the Drosophila germline stem cell. Development 130: 11591170 .

Chen, D. and McKearin, D. 2005. Gene circuitry controlling a stem cell niche. Curr. Biol. 15: 179-184.

Cox, D.N., Chao, A., Baker, J., Chang, L., Qiao, D., and Lin, H. 1998. A novel class of evolutionarily conserved genes defined by piwi are essential for stem cell self-renewal. Genes Dev. 12: 3715-3727.

Cox, D.N., Chao, A., and Lin, H. 2000. piwi encodes a nucleoplasmic factor whose activity modulates the number and division rate of germline stem cells. Development 127: 503-514.

DasGupta, R. and Fuchs, E. 1999. Multiple roles for activated LEF/TCF transcription complexes during hair follicle development and differentiation. Development 126: 4557-4568.

Decotto, E. and Spradling, A.C. 2005. The Drosophila ovarian and testis stem cell niches: Similar somatic stem cells and signals. Dev. Cell 9: 501-510.

Deuring, R., Fanti, L., Armstrong, J.A., Sarte, M., Papoulas, O., Prestel, M., Daubresse, G., Verardo, M., Moseley, S.L., Berloco, M., et al. 2000. The ISWI chromatin-remodeling protein is required for gene expression and the maintenance of higher order chromatin structure in vivo. Mol. Cell 5: 355-365.

Eberhart, C.G. and Wasserman, S.A. 1995. The pelota locus encodes a protein required for meiotic cell division: An analysis of G2/M arrest in Drosophila spermatogenesis. Development 121: 3477-3486.

Forbes, A. and Lehmann, R. 1998. Nanos and Pumilio have critical roles in the development and function of Drosophila germline stem cells. Development 125: 679-690.
Forbes, A.J., Lin, H., Ingham, P.W., and Spradling, A.C. 1996. hedgehog is required for the proliferation and specification of ovarian somatic cells prior to egg chamber formation in Drosophila. Development 122: 1125-1135.

Gilboa, L. and Lehmann, R. 2004. Repression of primordial germ cell differentiation parallels germ line stem cell maintenance. Curr. Biol. 14: 981-986.

Hatfield, S.D., Shcherbata, H.R., Fischer, K.A., Nakahara, K., Carthew, R.W., and Ruohola-Baker, H. 2005. Stem cell division is regulated by the microRNA pathway. Nature 435: 974-978.

Haug, J.S., He, X.C., Grindley, J.C., Wunderlich, J.P., Gaudenz, K., Ross, J.T., Paulson, A., Wagner, K.P., Xie, Y., Zhu, R., et al. 2008. N-cadherin expression level distinguishes reserved versus primed states of hematopoietic stem cells. Cell Stem Cell 2: $367-379$.

Jin, Z. and Xie, T. 2007. Dcr-1 maintains Drosophila ovarian stem cells. Curr. Biol. 17: 539-544.

Jin, Z., Kirilly, D., Weng, C., Kawase, E., Song, X., Smith, S., Schwartz, J., and Xie, T. 2008. Differentiation-defective stem cells outcompete normal stem cells for niche occupancy in the Drosophila ovary. Cell Stem Cell 2: 39-49.

Kai, T. and Spradling, A. 2003. An empty Drosophila stem cell niche reactivates the proliferation of ectopic cells. Proc. Natl. Acad. Sci. 100: 4633-4638.

Kai, T., Williams, D., and Spradling, A.C. 2005. The expression profile of purified Drosophila germline stem cells. Dev. Biol. 283: 486-502.

Kawase, E., Wong, M.D., Ding, B.C., and Xie, T. 2004. Gbb/Bmp signaling is essential for maintaining germline stem cells and for repressing bam transcription in the Drosophila testis. Development 131: 1365-1375.

King, F.J. and Lin, H. 1999. Somatic signaling mediated by $f_{s}(1) Y b$ is essential for germline stem cell maintenance during Drosophila oogenesis. Development 126: 1833-1844.

King, F.J., Szakmary, A., Cox, D.N., and Lin, H. 2001. Yb modulates the divisions of both germline and somatic stem cells through piwi- and $h$ h-mediated mechanisms in the Drosophila ovary. Mol. Cell 7: 497-508.

Kirilly, D., Spana, E.P., Perrimon, N., Padgett, R.W., and Xie, T. 2005. BMP signaling is required for controlling somatic stem cell self-renewal in the Drosophila ovary. Dev. Cell 9: 651662.

Korinek, V., Barker, N., Morin, P.J., van Wichen, D., de Weger, R., Kinzler, K.W., Vogelstein, B., and Clevers, H. 1997. Constitutive transcriptional activation by a $\beta$-catenin-Tcf complex in $\mathrm{APC}^{-/-}$colon carcinoma. Science 275: 1784-1787.

Korinek, V., Barker, N., Moerer, P., van Donselaar, E., Huls, G., Peters, P.J., and Clevers, H. 1998. Depletion of epithelial stemcell compartments in the small intestine of mice lacking Tcf4. Nat. Genet. 19: 379-383.

Kretzschmar, M. and Massagué, J. 1998. SMADs: Mediators and regulators of TGF- $\beta$ signaling. Curr. Opin. Genet. Dev. 8: 103-111.

Lee, H.H., Kim, Y.S., Kim, K.H., Heo, I., Kim, S.K., Kim, O., Kim, H.K., Yoon, J.Y., Kim, H.S., Kim do, J., et al. 2007. Structural and functional insights into Dom34, a key component of no-go mRNA decay. Mol. Cell 27: 938-950.

Li, L. and Xie, T. 2005. Stem cell niche: Structure and function. Annu. Rev. Cell Dev. Biol. 21: 605-631.

Lin, H. and Spradling, A.C. 1997. A novel group of pumilio mutations affects the asymmetric division of germline stem cells in the Drosophila ovary. Development 124: 2463-2476.

Maines, J.Z., Park, J.K., Williams, M., and McKearin, D.M. 2007. Stonewalling Drosophila stem cell differentiation by epigenetic controls. Development 134: 1471-1479.

Margolis, J. and Spradling, A. 1995. Identification and behavior of epithelial stem cells in the Drosophila ovary. Development 121: 3797-3807.

McKearin, D.M. and Ohlstein, B. 1995. A role for the Drosophila Bag-of-marbles protein in the differentiation of cystoblasts from germline stem cells. Development 121: 2937-2947.

McKearin, D.M. and Spradling, A.C. 1990. bag-of-marbles: A Drosophila gene required to initiate both male and female gametogenesis. Genes Dev. 4: 2242-2251.

Morrison, S.J. and Spradling, A.C. 2008. Stem cells and niches: 
Mechanisms that promote stem cell maintenance throughout life. Cell 132: 598-611.

Nystul, T. and Spradling, A. 2007. An epithelial niche in the Drosophila ovary undergoes long-range stem cell replacement. Cell Stem Cell 1: 277-285.

Ohlstein, B. and McKearin, D. 1997. Ectopic expression of the Drosophila Bam protein eliminates oogenic germline stem cells. Development 124: 3651-3662.

Ohlstein, B., Lavoie, C.A., Vef, O., Gateff, E., and McKearin, D.M. 2000. The Drosophila cystoblast differentiation factor, benign gonial cell neoplasm, is related to DExH-box proteins and interacts genetically with bag-of-marbles. Genetics 155: 1809-1819.

Pan, L., Chen, S., Weng, C., Call, G.B., Zhu, D., Tang, H., Zhang, N., and Xie, T. 2007. Stem cell aging is controlled both intrinsically and extrinsically in the Drosophila ovary. Cell Stem Cell 1: 458-469.

Park, J.K., Liu, X., Strauss, T.J., McKearin, D.M., and Liu, Q. 2007. The miRNA pathway intrinsically controls self-renewal of Drosophila germline stem cells. Curr. Biol. 17: 533-538.

Song, X. and Xie, T. 2002. DE-cadherin-mediated cell adhesion is essential for maintaining somatic stem cells in the Drosophila ovary. Proc. Natl. Acad. Sci. 99: 14813-14818.

Song, X. and Xie, T. 2003. wingless signaling regulates the maintenance of ovarian somatic stem cells in Drosophila. Development 130: 3259-3268.

Song, X., Zhu, C.H., Doan, C., and Xie, T. 2002. Germline stem cells anchored by adherens junctions in the Drosophila ovary niche. Science 296: 1855-1857.

Song, X., Wong, M.D., Kawase, E., Xi, R., Ding, B.C., McCarthy, J.J., and Xie, T. 2004. Bmp signals from niche cells directly repress transcription of a differentiation-promoting gene, bag of marbles, in germline stem cells in the Drosophila ovary. Development 131: 1353-1364.

Song, X., Call, G.B., Kirilly, D., and Xie, T. 2007. Notch signaling controls germline stem cell niche formation in the
Drosophila ovary. Development 134: 1071-1080.

Szakmary, A., Cox, D.N., Wang, Z., and Lin, H. 2005. Regulatory relationship among piwi, pumilio, and bag-of-marbles in Drosophila germline stem cell self-renewal and differentiation. Curr. Biol. 15: 171-178.

Tower, J. 2000. Transgenic methods for increasing Drosophila life span. Mech. Ageing Dev. 118: 1-14.

Valencia-Sanchez, M.A., Liu, J., Hannon, G.J., and Parker, R. 2006. Control of translation and mRNA degradation by miRNAs and siRNAs. Genes Dev. 20: 515-524.

Wang, Z. and Lin, H. 2004. Nanos maintains germline stem cell self-renewal by preventing differentiation. Science 303: 2016 2019.

Wang, Z. and Lin, H. 2005. The division of Drosophila germline stem cells and their precursors requires a specific cyclin. Curr. Biol. 15: 328-333.

Ward, E.J., Shcherbata, H.R., Reynolds, S.H., Fischer, K.A., Hatfield, S.D., and Ruohola-Baker, H. 2006. Stem cells signal to the niche through the Notch pathway in the Drosophila ovary. Curr. Biol. 16: 2352-2358.

Xi, R. and Xie, T. 2005. Stem cell self-renewal controlled by chromatin remodeling factors. Science 310: 1487-1489.

Xi, R., Doan, C., Liu, D., and Xie, T. 2005. Pelota controls selfrenewal of germline stem cells by repressing a Bam-independent differentiation pathway. Development 132: 5365-5374.

Xie, T. and Spradling, A.C. 1998. decapentaplegic is essential for the maintenance and division of germline stem cells in the Drosophila ovary. Cell 94: 251-260.

Xie, T. and Spradling, A.C. 2000. A niche maintaining germ line stem cells in the Drosophila ovary. Science 290: 328-330.

Zhang, Y. and Kalderon, D. 2001. Hedgehog acts as a somatic stem cell factor in the Drosophila ovary. Nature 410: 599-604.

Zhu, C.H. and Xie, T. 2003. Clonal expansion of ovarian germline stem cells during niche formation in Drosophila. Development 130: 2579-2588. 


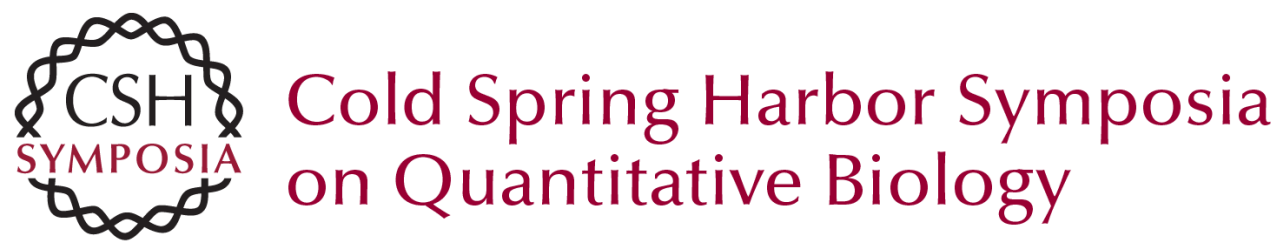

\section{Interactions between Stem Cells and Their Niche in the Drosophila Ovary}

T. Xie, X. Song, Z. Jin, et al.

Cold Spring Harb Symp Quant Biol 2008 73: 39-47 originally published online November 6, 2008 Access the most recent version at doi:10.1101/sqb.2008.73.014

References This article cites 56 articles, 30 of which can be accessed free at: http://symposium.cshlp.org/content/73/39.full.html\#ref-list-1

\section{License}

Email Alerting Receive free email alerts when new articles cite this article - sign up in the box at the Service top right corner of the article or click here. 